Hanif M. Ladak and W. Robert J. Funnell

\title{
Finite-element modeling of the normal and surgically repaired cat middle ear
}

Published in:

Journal of the Acoustical Society of America, 100(2), pt. 1, August 1996, pp. 933-944

Copyright (1996) Acoustical Society of America. This article may be downloaded for personal use only. Any other use requires prior permission of the author and the Acoustical Society of America.

doi:10.1121/1.416205

http://scitation.aip.org/content/asa/journal/jasa/100/2/10.1121/1.416205 


\title{
Finite-element modeling of the normal and surgically repaired cat middle ear
}

\author{
Hanif M. Ladak and W. Robert J. Funnell \\ Department of BioMedical Engineering, McGill University, 3775, rue University, Montréal, Québec H3A \\ 2B4, Canada
}

(Received 28 February 1995; accepted for publication 3 March 1996)

\begin{abstract}
In this work, three-dimensional finite-element models of the normal and surgically repaired cat middle ear were developed. The normal middle-ear model was formed by adding explicit representations for the footplate and cochlear load to an existing model of the cat eardrum. The footplate was modeled as a thin plate with a thickened rim. The cochlear load was represented by springs attached along the footplate's periphery. The model is valid for frequencies below $1 \mathrm{kHz}$ and for physiological sound levels. Eardrum and manubrium displacements, and out-of-plane displacements of the footplate's center, were found to compare well with experimental results. The normal model was modified to simulate the effects of two types of middle-ear surgery, both of which are used to repair a discontinuous ossicular chain. Bulging of the footplate was found to occur when a prosthesis made direct contact with the footplate. The location of the prosthesis along the manubrium did not affect the motion of the footplate as long as the joints were all rigid. When the joints were flexible, the largest displacements occurred when the prosthesis was positioned near the upper end of the manubrium. (C) 1996 Acoustical Society of America.
\end{abstract}

PACS numbers: 43.64.Bt, 43.64.Ha [RAS]

\section{INTRODUCTION}

Discontinuity of the middle-ear ossicular chain results in conductive hearing loss. The most common type of ossicular discontinuity is caused by loss of the incudal long process; the second most common type is caused by loss of both the incudal long process and the stapes superstructure (Austin, 1971). When the incudal long process alone is missing, a prosthesis (e.g., ossicular bone graft) can be fitted between the manubrium of the malleus and the head of the stapes; the resulting structure is called a malleus-stapes assembly (MSA). When both the incudal long process and the stapes superstructure are missing, a prosthesis can be fitted between the manubrium and the stapedial footplate; this structure is called a malleus-footplate assembly (MFA). The MSA and the MFA do not result in the complete elimination of hearing loss (e.g., Vartiainen and Nuutinen, 1992). A quantitative understanding of the mechanics of the normal and surgically repaired middle ear should elucidate some of the reasons for this failure and would aid in the design of middle-ear prostheses.

A few models of the normal and surgically repaired middle ear have been developed. Wada et al. (1992) developed a finite-element model of the human middle ear. Although finite-element models of the cat eardrum have been developed (Funnel and Laszlo, 1978; Funnell, 1983; Funnell et al., 1987, 1992), no models of the entire cat middle ear exist. Finite-element models of the surgically repaired middle ear have also been reported. Lesser et al. (1991) developed a two-dimensional model of the MSA in humans. Their model is oversimplified since the eardrum and middle ear are three dimensional and do not possess the type of symmetry required to permit a two-dimensional representation. Williams and Lesser (1992) have developed a threedimensional model of the Fisch II Spandrel prosthesis in the human middle ear. (The Fisch II Spandrel prosthesis is similar to the MFA.) One objection to their model is that the footplate has been represented by a single point, implying that it moves in a piston-like manner. It is possible, however, for the footplate to tilt in the surgically repaired middle ear depending on the placement of the prosthesis, and tilting of the footplate may affect volume displacements of the cochlear fluids and hence hearing sensitivity (Vlaming and Feenstra, 1986).

Other methods have also been used to model the surgically repaired middle ear. Wada et al. (1990) used analytical techniques to model an artificial auditory ossicle. Their model assumes a flat, circular eardrum; presumably, this was done to make the analyses tractable. However, the results of Funnell and Laszlo (1978) indicate that the threedimensional shape of the eardrum is very important to its function. Peake et al. (1992) developed a lumped-parameter model of type IV tympanoplasty, a surgical procedure used to shield the round window from acoustic stimulation when the eardrum, malleus and incus are missing. This technique is not in any way similar to the MSA or the MFA.

This paper presents a finite-element model of the normal cat middle ear which has been developed by adding explicit representations of the footplate and cochlear load to an existing model of the cat eardrum (Funnell et al., 1987). The model was then modified to investigate the effects of middleear surgery. The models simulate the behaviour of the normal and surgically repaired middle ear in response to uniform pressures of low enough frequencies that inertial and damping effects may be ignored. This corresponds to frequencies below about $1 \mathrm{kHz}$. The models are restricted to sound pressures low enough that the responses of the middleear structures and of the prosthesis are linear.

Section I describes the models and the mechanical prop- 

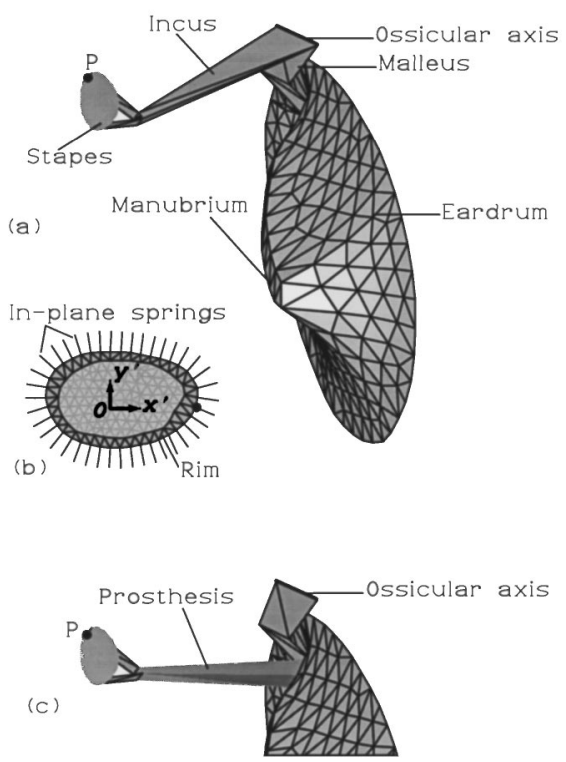

FIG. 1. Geometry of the normal middle-ear and MSA models. (a) View of middle-ear model showing medial aspect of the eardrum. The footplate mesh and the cochlear load are not shown for clarity. (b) Footplate model showing in-plane springs. The out-of-plane springs are not shown but would be perpendicular to the plane of the paper. The $x^{\prime}-y^{\prime}$ axes are shown along with the origin $O$. These axes will be referred to in Sec. II. (c) Top portion of MSA model showing prosthesis. The posterior $(\mathrm{P})$ end of the footplate is indicated by a dot.

erties that we have assumed. Section II presents results describing the behavior of the models and the effects of variations of several parameters and of prosthesis location.

\section{FINITE-ELEMENT MODELS}

\section{A. Normal middle-ear model}

Figure 1(a) shows the normal middle-ear model which includes the eardrum, the ossicles and the cochlear load. The eardrum model is equivalent to previous models (Funnell, 1983; Funnell et al., 1987). The eardrum is modeled as a linearly elastic thin shell, the material of which is assumed to be homogeneous and isotropic. The material properties of the eardrum are based on a review of the literature (Funnell and Laszlo, 1982). The part of the model corresponding to the pars tensa has a Young's modulus, or stiffness, of $2 \times 10^{8}$ dyn $\mathrm{cm}^{-2}$, an overall thickness of $40 \mu \mathrm{m}$ and a Poisson's ratio of 0.3 . The pars flaccida is modeled as having a Young's modulus of $1 \times 10^{6} \mathrm{dyn}^{-2}$, a thickness of $40 \mu \mathrm{m}$ and a Poisson's ratio of 0.3 . The portion of the annular ligament separating the pars tensa from the pars flaccida has a Young's modulus and a Poisson's ratio identical to those of the pars tensa but a thickness of $300 \mu \mathrm{m}$. The mesh of triangular elements representing the eardrum has a "nominal resolution"' (Funnell, 1983) of 15 elements/diameter. The overall three-dimensional curvature of the eardrum is expressed by a "normalized radius of curvature" of 1.19 (Funnell, 1983).

The ossicles are modeled as homogeneous, isotropic bone having a Young's modulus of $2 \times 10^{11} \mathrm{dyn}^{-2}$ and a Poisson's ratio of 0.3 (Funnell et al., 1992). With the exception of the stapedial footplate, the ossicles are modeled as
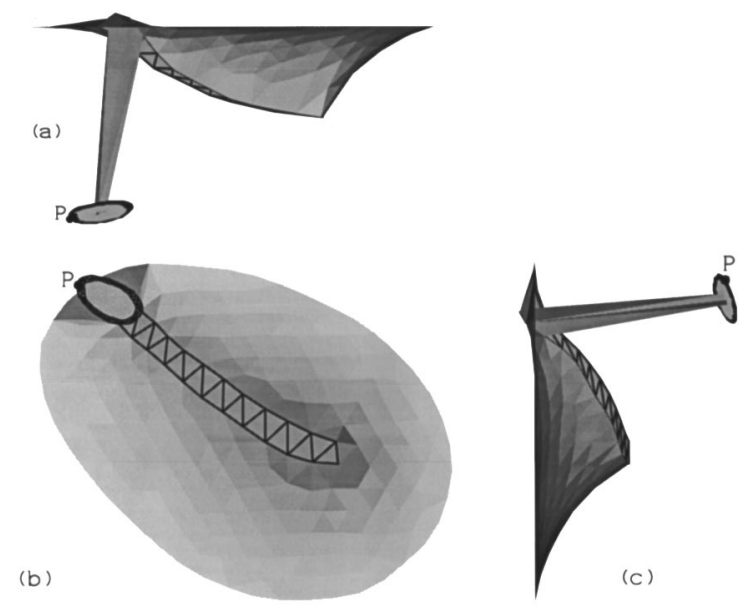

FIG. 2. Three orthogonal views of the MFA model showing location and orientation of the footplate. (a) View along antero-posterior direction. (b) Medial view along an axis perpendicular to the tympanic ring. (c) View along infero-superior direction. The posterior $(\mathrm{P})$ end of the footplate is indicated by a dot.

being so thick as to be effectively rigid. Five elements representing the neck and head of the malleus couple the manubrium to the ossicular axis of rotation as shown in Fig. 1(a). Four elements, representing the long process of the incus, connect the axis of rotation to the tops of the stapedial crura. Each crus is modeled with two plate elements. The shapes of the elements representing the malleus, incus, and stapedial crura are not important since they are thick enough to be effectively rigid and since the frequencies considered here are low enough that there are no inertial effects.

The incudomallear joint appears to be effectively rigid for physiological sound-pressure levels and for frequencies below $3000 \mathrm{~Hz}$ (Guinan and Peake, 1967; Vlaming, 1987), and is modeled here as being rigid. The incudostapedial joint appears to be effectively rigid in the medio-lateral direction for physiological sound-pressure levels (Guinan and Peake, 1967; Vlaming, 1987), but in experiments involving contractions of the stapedius muscle the joint does permit displacements of the stapes in the antero-posterior direction relative to the incus (Pang and Peake, 1986). Since the forces developed in the stapedius were not measured in those experiments, it is not clear whether the joint would be flexible in this direction for physiological sound-pressure levels for which the present model is valid. For simplicity, therefore, the joint is modelled here as being rigid in all directions.

The mesh for the footplate, shown in Fig. 1(b), has a nominal resolution of 15 elements/diameter. (The diameter of the footplate is smaller than that of the eardrum, so the elements of the footplate mesh are smaller than those of the eardrum mesh.) The shape and size of the footplate were obtained from a micrograph (Guinan and Peake, 1967, Fig. 11). The location and orientation of the footplate were determined from a computer reconstruction of the cat middle ear (Funnell, 1989; Funnell et al., 1992) and are shown in Fig. 2. The thickness of the rim of the footplate has been taken to be $200 \mu \mathrm{m}$; that of the central portion has been taken to be 20 $\mu \mathrm{m}$ (Guinan and Peake, 1967, Fig. 11). 
The load due to the ligaments of the ossicular chain is represented by an effective angular stiffness of $10^{4}$ dyn $\mathrm{cm}$ about a fixed axis of rotation. This value was obtained by subtracting the value for the angular stiffness of the cochlea $\left(18 \times 10^{3}\right.$ dyn $\left.\mathrm{cm}\right)$ given by Funnell and Laszlo (1978) from the total angular stiffness about the axis in their model $\left(28 \times 10^{3} \mathrm{dyn} \mathrm{cm}\right)$; the resultant stiffness represents contributions due to the posterior incudal ligament and the anterior mallear process. The cochlear load in the present model is represented in a different manner as discussed below. The ossicular axis of rotation is assumed to be fixed, an assumption that appears to be acceptable for low frequencies (Guinan and Peake, 1967; Decraemer et al., 1991). As in earlier models, the axis is taken to lie in the plane of the tympanic ring. All degrees of freedom on the axis are constrained to be zero except for rotation about it.

The cochlear load acting upon the footplate is stiffnessdominated for frequencies below $300 \mathrm{~Hz}$ (Lynch et al., 1982). The stiffness is mainly due to the annular ligament and is represented in our model by springs distributed around the periphery of the footplate. These springs constrain both the out-of-plane and in-plane displacements of the footplate. The springs constraining the footplate's out-of-plane motion are perpendicular to the plane of the footplate; one spring is attached to each of the evenly spaced nodes around the periphery of the footplate. Lynch et al. (1982) have given a value of $0.36 \times 10^{-9} \mathrm{~cm}^{5} \mathrm{dyn}^{-1}$ for the acoustic compliance of the stapediocochlear complex which, for a footplate of area $1.26 \mathrm{~mm}^{2}$, is equivalent to a mechanical stiffness of $4.4 \times 10^{5} \mathrm{dyn} \mathrm{cm}^{-1}$ divided evenly among the uniformly spaced springs around the outside of the footplate.

The in-plane stiffness of the annular ligament is represented by a second set of springs, perpendicular to the rim of the footplate but in its plane; once again, one such spring is attached to each of the external nodes of the footplate. Assuming that the mechanically important part of the annular ligament is contained in the annular space surrounding the footplate, and assuming that the material of the ligament is unstressed, isotropic, homogeneous and uniformly distributed, Lynch et al. (1982) estimated the Young's modulus of the annular ligament to be $10^{5} \mathrm{dyn}^{-2}$. As noted by them, this value is low compared to that of other ligaments; however, as not enough data are available on the properties of the ligament, the above value has been adopted in calculating the in-plane stiffness of the annular ligament in the present model. A portion of the annular ligament between two adjacent external nodes can be considered to be a slab of uniform rectangular cross-sectional area with its stiffness given by $k_{i}=E t l_{i} / w$, where $E$ is the Young's modulus of the annular ligament, $l_{i}$ is the distance between adjacent nodes, and $t$ $(200 \mu \mathrm{m})$ and $w(20 \mu \mathrm{m})$ are the thickness and width, respectively. The dimensions of the annular ligament are assumed to be constant around its perimeter (Guinan and Peake, 1967, Fig. 11). The stiffness of each segment of the annular ligament was computed and divided equally between the springs at the two nodes. Note that shearing due to in-plane rotation of the footplate is ignored.
Not represented in the models are the effects of the middle-ear cavities; this is equivalent to an experimental situation in which the middle-ear cavities and septum are open. The tensor tympani and the stapedius muscle are also not modeled, approximately simulating the relaxed state of the middle-ear muscles in temporal bone preparations and in anesthetized animals.

This model as well as those of the MSA and MFA were implemented using SAP IV, a structural finite-element analysis program for computing the response of linear systems (Bathe et al., 1974).

\section{B. MSA model}

It is assumed that the existing middle-ear structures being modeled have not been damaged by middle-ear disease or by the surgical procedure. Thus the MSA model, as shown in Fig. 1(c), is identical to the normal middle-ear model except that the four plate elements corresponding to the incudal long process are replaced by a single brick element representing the prosthesis. (A single element is adequate because the prosthesis is considered to be rigid.) Whereas the plate elements representing the incus in the normal model connect the stapes to the mallear head, the brick in the MSA model connects the stapes to the manubrium of the malleus. In the present model, an eight-node brick element with three translational degrees of freedom at each node has been used. The Young's modulus and Poisson's ratio of the prosthesis are the same as those of the other bones in this model; since the prosthesis is effectively rigid, it could equally well model rigid synthetic materials such as ceramic. The prosthesis is connected between a quadrilateral region on the manubrium and the tops of the crura; the quadrilateral region on the manubrium is defined by the manubrial mesh. The prosthesis is connected to the uppermost quadrilateral region of the manubrial mesh except in those simulations intended to investigate variations of prosthesis position (see Sec. II E). When the brick element is used to model the prosthesis, the joints between the prosthesis and bones are assumed to be inflexible. In some simulations, a truss element is used for the prosthesis in order to model "pin" joints (see Sec. II E). This truss element has a Young's modulus and crosssectional area high enough to make it effectively rigid.

In the MSA model, it is necessary to use two thin-shell elements to bridge the gap between the tops of the crura in order to constrain the normal rotational degree of freedom of the thin-shell elements representing the crura. The normal rotational degree of freedom for the thin-shell element is not defined and must be constrained (Bathe et al., 1974). These last elements were not required in the normal middle-ear model since the thin-shell elements representing the incudal long process provide the required constraints.

Another modification in some of the simulations (see Sec. II B and C) involving the MSA and the MFA was the removal of the axis of rotation and of the five elements representing the neck and head of the malleus in order to simulate the effects of surgery: the neck and head of the malleus are sometimes removed during surgery, thus destroying the axis of rotation. 


\section{MFA model}

As shown in Fig. 2, the MFA model differs from the MSA model in that (1) the crura are removed, and (2) the prosthesis makes direct contact with the footplate. The medial end of the prosthesis is attached to a square region of area $0.04 \mathrm{~mm}^{2}$ at the center of the footplate mesh.

\section{Range of validity}

The normal cat middle ear has been shown to be linear for sound pressure levels up to at least $130 \mathrm{~dB}$ SPL for frequencies below $1500 \mathrm{~Hz}$ (Guinan and Peake, 1967). It is reasonable to assume that the reconstructed middle ear also behaves linearly for these sound levels. The acoustic stimulus in the present models is represented by a uniformly distributed pressure of $100 \mathrm{~dB}$ SPL applied to the lateral surface of the eardrum. A linear model is thus sufficient for the sound-pressure level used in this study.

The range of displacements for which the models are valid is primarily governed by a constraint common to thinplate and thin-shell elements: The displacements must be small compared with the thickness of the structure in order to permit the assumption that in-plane and out-of-plane displacements are not coupled. A check on the validity of the model is provided by ascertaining that displacements are a small fraction of the thickness. The largest eardrum displacements are less than $2 \%$ of its thickness, and the largest footplate displacements are less than $1 \%$ of its thickness.

The upper frequency for which the models are valid is limited by the omission of damping and inertial effects. Exclusion of these effects in the models makes their behavior independent of frequency. For the stapediocochlear system alone, damping and inertia have some effects even at frequencies as low as $300 \mathrm{~Hz}$ (Lynch et al., 1982). However, the behavior of the intact cat middle ear appears to be approximately constant up to about $1 \mathrm{kHz}$ (Guinan and Peake, 1967); therefore, it is permissible to compare displacements calculated using the normal middle-ear model with experimental data up to $1 \mathrm{kHz}$. Presumably, damping and inertial effects introduced by the prosthesis in the MSA and MFA are also negligible up to $1 \mathrm{kHz}$.

\section{E. Adequacy of mesh resolution}

The resolutions of the eardrum and footplate meshes are specified in terms of a "nominal resolution" which is used by the automatic mesh generator as a target for the number of elements across the diameter of the eardrum or footplate, respectively. The effects of these mesh resolutions on displacements were tested separately.

The resolution of the eardrum mesh (without an ossicular axis) was varied from 6 to 20 elements/diameter. The load due to the prosthesis and cochlea present in the MFA and MSA models was represented by two springs attached to the upper end of the manubrium. For mesh resolutions of 12 elements/diameter and greater, variations in the maximum drum displacement and umbo displacement were found to be less than $4 \%$. Thus a resolution of 15 elements/diameter for the eardrum is adequate.
To investigate the effect of mesh resolution on deformation of the footplate, the footplate was fully clamped around its periphery to eliminate rigid-body motion. Concentrated loads of 1 dyn were applied to the four corners of the square at the center of the footplate, which serves as the attachment site for the prosthesis. The resolution of the footplate mesh was varied from 6 to 20 elements/diameter. For resolutions of 12 elements/diameter and greater, the maximum displacement varied less than 3\%. Thus a resolution of 15 elements/ diameter for the footplate mesh is again adequate.

In order to test the effects of mesh resolution on the overall structure, two MFA models without an ossicular axis were generated. In the first model, both the eardrum and footplate had resolutions of 15 elements/diameter. In the second model, both had resolutions of 20 elements/diameter. The maximum drum displacement calculated using the first model was within $2.6 \%$ of the value computed using the second model; the umbo displacement was within $0.8 \%$; and footplate displacements were within $0.2 \%$. This confirms that a model with eardrum and footplate resolutions of 15 elements/diameter is adequate.

\section{RESULTS}

\section{A. Results for the normal middle-ear model}

Displacement patterns calculated for the eardrum in the normal middle-ear model are qualitatively similar to the lowfrequency patterns observed experimentally by Khanna (1970) and to the patterns determined using a previous model (Funnell et al., 1987). The maximal drum displacement occurs in the posterior region of the pars tensa. It has a magnitude of $484 \mathrm{~nm}$ for a low-frequency pure-tone input of 100 dB SPL. Tonndorf and Khanna (1971) reported a maximal drum displacement of $1500 \mathrm{~nm}$ at $600 \mathrm{~Hz}$ and $111 \mathrm{~dB}$ SPL; this is equivalent to $420 \mathrm{~nm}$ at $100 \mathrm{~dB}$ SPL. The maximal drum displacement calculated using the present model is $15 \%$ higher than what they found. The present value is, however, 27\% lower than that calculated by Funnell et al. (1987), primarily because of the different way in which the ossicular and cochlear loads were modeled by them. Note that, as specified by Funnell et al. (1992), the ossicular stiffness in the previous models was $14 \mathrm{kdyn} \mathrm{cm}$ (rather than the $28 \mathrm{kdyn} \mathrm{cm}$ as given in Funnell (1983) and Funnell et al. (1987), due to an error in the implementation of the models in those papers). In the present model, the effective rotational stiffness about the fixed axis due to the ossicular and cochlear loads is $65.5 \mathrm{kdyn} \mathrm{cm}$ (i.e., about four times larger than in the previous models). The displacement decrease of $27 \%$ is thus consistent with the observation in Funnell and Laszlo (1978) that an increase in rotational stiffness by a factor of 2 results in a decrease in the maximal eardrum displacement of $11 \%$.

The umbo displacement calculated using the present model is $161 \mathrm{~nm}$. Tonndorf and Khanna (1971) reported the ratio of peak drum displacement to umbo displacement to be 2.2; this corresponds to an umbo displacement of $191 \mathrm{~nm}$. The model predicts a value that is $16 \%$ lower than what they found. Funnell et al. (1987) computed an umbo displacement of $245 \mathrm{~nm}$ using their model. The value calculated using the 

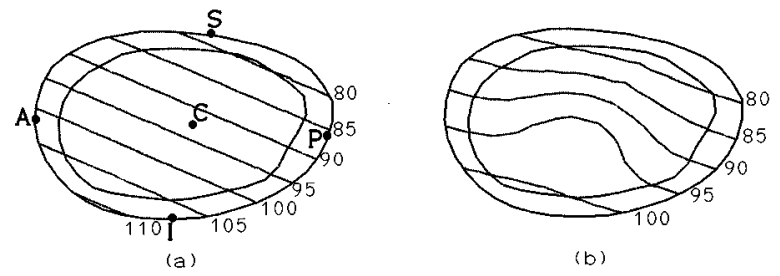

FIG. 3. Iso-amplitude contours for out-of-plane component of footplate displacement calculated for a pure-tone input of $100 \mathrm{~dB}$ SPL using (a) the normal middle-ear model and (b) the MFA model with an intact ossicular axis. All displacements are in nanometres. The anterior (A), central (C), inferior $(\mathrm{I})$, posterior $(\mathrm{P})$, and superior $(\mathrm{S})$ points are indicated in part (a).

present model is $34 \%$ lower than that calculated by them; as expected, the difference in the representation of the ossicular and cochlear loads has an even stronger effect on umbo displacement than on eardrum displacement.

The ratio of maximal eardrum displacement to umbo displacement is one quantitative measure for comparing the spatial pattern of the model with experiment. The value computed using the present model is 3.0, which is $36 \%$ higher than that given by Tonndorf and Khanna (1971). The present result is $11 \%$ higher than the value computed by Funnell et al. (1987).

It is convenient to describe the three-dimensional motion of the footplate by decomposing this motion into components within the plane of the footplate and a component normal to its plane. The description of footplate motion in terms of these components has some physiological significance: the normal (out-of-plane) component of footplate motion causes displacement of the cochlear fluid, whereas in-plane components are not effective in displacing this fluid.

Figure 3(a) shows contours for the out-of-plane component of footplate displacement. The displacements are 102 $\mathrm{nm}$ for the anterior end [point A in Fig. 3(a)], $93 \mathrm{~nm}$ for the center (point $\mathrm{C}$ ), and $85 \mathrm{~nm}$ for the posterior end (point $\mathrm{P}$ ). The displacement of the central point was used by Vlaming and Feenstra (1986) to compare the mechanics of the footplate in various cadaver preparations. If the footplate is perfectly rigid, then the out-of-plane component of displacement of this point, when multiplied by the area of the footplate, gives the volume displacement of the cochlear fluids; on the other hand, if the footplate deforms or bulges at the center, then this product will overestimate the volume displacement of the cochlear fluids. Any bulging of the footplate that might occur at its center can be quantified using the bulge ratio $\beta=\left[w_{c}-\frac{1}{2}\left(w_{A}+w_{P}\right)\right] / w_{c}$, where $w_{A}, w_{C}$, and $w_{P}$ are the out-of-plane components of displacement at the anterior, central, and posterior points of the footplate, respectively. If the footplate is rigid, then the displacement of the central point will be equal to the average of the displacements of the anterior and posterior points and the bulge ratio will be zero; this is more or less the case for the present model since the bulge ratio has the very small value of 0.011 . The bulge ratio should be exactly zero in this model since no forces are applied to the center of the footplate; however, it is slightly nonzero since the "central", point defined by the finite-element mesh is not exactly halfway between the anterior and posterior ends.
The difference in displacement amplitude between the anterior and posterior ends indicates that the footplate tilts. The amount of antero-posterior tilting relative to the displacement of the center can be characterized by the anteroposterior tilt ratio $\Delta_{A P}=\left(w_{A}-w_{P}\right) / w_{C}$. The anteroposterior tilt ratio is 0.18 for this model, indicating that the difference between the displacements of the two ends is $18 \%$ as large as the displacement of the center of the footplate; a positive ratio indicates that the anterior end displaces more than the posterior end. Tilting of the footplate also results in differences in the displacements of the inferior and superior edges [I and $S$, respectively, in Fig. 3(a)]. An infero-superior tilt ratio may be calculated as $\Delta_{I S}=\left(w_{I}-w_{S}\right) / w_{C}$, where $w_{I}$ and $w_{S}$ are the out-of-plane components of displacement of the inferior and superior edges, respectively. The displacement of the superior edge is $78 \mathrm{~nm}$ and that of the inferior edge is $108 \mathrm{~nm}$, giving an infero-superior tilt ratio of 0.33 .

The in-plane component of footplate motion can be decomposed into $x^{\prime}$ and $y^{\prime}$ components; the $x^{\prime}$ and $y^{\prime}$ axes are attached to the to the footplate as shown in Fig. 1(b). Displacement patterns for the $x^{\prime}$ and $y^{\prime}$ components consist of evenly spaced horizontal and vertical lines, respectively; hence, it can be concluded that the in-plane motion of the footplate is that of a rigid body. In order to quantify this conclusion, a rigid-body translation and rotation were fitted to the finite-element results as described below; nodal displacements corresponding to this rigid-body motion were then compared with the nodal displacements calculated using the finite-element software. The finite-element results in general will include displacements due to both deformation and rigid-body motion.

To estimate the rigid-body translation and rotation, a point was arbitrarily chosen about which the angle of rotation was to be estimated. The point $O$ shown in Fig. 1 was chosen. The displacement of this point, calculated using the finite-element software, gives an estimate for the rigid-body translation of the footplate which, in this case, has an $x^{\prime}$ component of $68 \mathrm{~nm}$ and a $y^{\prime}$ component of $89 \mathrm{~nm}$. The rigid-body rotation can then be estimated using the formula $\theta \approx-\left(u_{i}-u_{j}\right) /\left(y_{i}-y_{j}\right) \approx-\left(v_{i}-v_{j}\right) /\left(x_{i}-x_{j}\right)$, where $u_{i}$ and $v_{i}$ are the $x^{\prime}$ and $y^{\prime}$ components, respectively, of displacement of node $i$ on the footplate, and $x_{i}$ and $y_{i}$ are the $x^{\prime}$ and $y^{\prime}$ coordinates of node $i$, respectively. In this case, the angle of rotation was found to be $9.0 \mu \mathrm{rad}$ in the counterclockwise direction. (The point labeled $O$ in Fig. 1 was chosen as node $i$, and the node directly above it was chosen as node $j$.)

In order to compare the magnitudes of displacements caused by deformation with those caused by rigid-body motion, the ratio $D / R$ was computed, where

$$
D=\frac{1}{n} \sum_{i=1}^{n} \sqrt{\left(u_{i}^{\prime}-u_{i}\right)^{2}+\left(v_{i}^{\prime}-v_{i}\right)^{2}}
$$

and

$$
R=\frac{1}{n} \sum_{i=1}^{n} \sqrt{\left(u_{i}^{\prime}\right)^{2}+\left(v_{i}^{\prime}\right)^{2}}
$$

where $u$ and $v$ again represent components of displacement in the $x^{\prime}$ and $y^{\prime}$ directions, respectively, and $n$ is the number 
of nodes in the footplate model. Unprimed quantities represent nodal displacements calculated using the finite-element software, and primed quantities represent nodal displacements calculated using the estimated rigid-body translation and rotation; the summations are over all of the nodes of the footplate. The quantity $D$ represents the average displacement amplitude due to deformation, whereas $R$ represents the average displacement amplitude due to rigid-body motion. For the footplate in the normal middle-ear model, the ratio $D / R$ was found to be $3.9 \times 10^{-4}$, indicating that the average displacement due to deformation is more than three orders of magnitude smaller than that due to rigid-body motion; it is thus reasonable to approximate the in-plane motion of the footplate as that of a rigid body.

It should be noted that the maximum in-plane displacement amplitude of the footplate is $116 \mathrm{~nm}$. This value is much smaller than the width of the annular ligament (20 $\mu \mathrm{m})$, thus justifying the assumption that the ligament behaves linearly.

The only detailed measurements of footplate motion in cats with which these results can be compared are those of Guinan and Peake (1967). These authors found that, to a first approximation, the footplate moves in a piston-like manner. Figure 14 of their paper indicates that the magnitude of footplate displacement per unit sound pressure at the eardrum is approximately $3.0 \times 10^{-7} \mathrm{~cm}^{3} \mathrm{dyn}^{-1}$, giving a stapes displacement of $85 \mathrm{~nm}$ for a pressure of $100 \mathrm{~dB}$ SPL (i.e., 28.28 dyn $\mathrm{cm}^{-2}$ ) applied to the eardrum; this is only $8 \%$ lower than the out-of-plane displacement at the center of the footplate calculated using the present model. As in the model, Guinan and Peake did not detect bulging of the footplate.

The results of Guinan and Peake indicate that tilting and in-plane motion of the footplate, if any, are small compared with the out-of-plane motion. The model, however, predicts tilting and in-plane motion with magnitudes similar to the out-of-plane of motion. Wada et al. (1992) have also reported large in-plane displacements of the footplate in their middle-ear model. They suggested that one reason for this type of motion may be that the incudostapedial joint in their model was assumed to be rigid. Similarly, in the present model the malleus and incus act as a single rigid body and the incudostapedial joint is also rigid. Therefore, rotation of the malleus and incus about the ossicular axis forces the stapes to rotate about the same axis, necessarily resulting in tilting and in-plane, as well as out-of-plane, motion of the footplate. A flexible incudostapedial joint may permit rotation of the incus and malleus about the ossicular axis without forcing the stapes to necessarily rotate about the same axis. More work needs to be done to estimate the stiffness of the incudostapedial joint and its effects on footplate motion.

\section{B. Results for MSA model}

Recall that for the MSA a prosthesis is placed between the manubrium and the stapedial head. The ossicular axis may or may not be intact, and results are reported here for both cases.

\section{With ossicular axis}

Displacement patterns and amplitudes for the eardrum and for the footplate calculated using the MSA model with an intact ossicular axis are all within $2 \%$ of those calculated for the normal middle-ear model. This similarity is to be expected since the ossicular chain in the normal model acts as a single rigid body that is, in effect, equivalent to the manubrium-prosthesis-stapes system in the MSA model.

\section{Without ossicular axis}

The maximum eardrum displacement calculated using the MSA model without the fixed ossicular axis is $644 \mathrm{~nm}$ which is approximately $30 \%$ larger than that calculated with the axis. Freeing the ossicular axis of rotation removes the constraints it places on the eardrum, thus permitting larger eardrum displacements. The umbo displacement is $327 \mathrm{~nm}$, or about twice as large as that with the ossicular axis. The displacement contours indicate that the manubrium rotates about an instantaneous axis having a very different position and orientation than the original fixed axis of rotation.

The out-of-plane component of displacement amplitude at the centre of the footplate is $127 \mathrm{~nm}$, approximately $36 \%$ larger than that calculated with the axis. The larger footplate displacement indicates better coupling of sound pressure to the cochlea. The anterior end of the footplate has a larger displacement amplitude than the posterior end, giving an antero-posterior tilt ratio of 0.55 , approximately three times as large as that for the previous models. The inferior edge has a slightly larger displacement amplitude than the superior edge; the infero-superior tilt ratio is 0.073 , about $22 \%$ as large as that for the previous models. The footplate in this model does not bulge significantly, having a bulge ratio of $1.5 \times 10^{-3}$.

Once again, the in-plane motion of the footplate can be described by a rigid-body motion. The $x^{\prime}$ component of translation is $63 \mathrm{~nm}$, similar to that calculated for the previous models; the $y^{\prime}$ component of translation has changed sign, to $-87 \mathrm{~nm}$. The angle of rotation is $-0.15 \mu \mathrm{rad}$, i.e., in the clockwise direction.

\section{Experimental data}

No measurements of footplate displacements have been made in cats with surgically repaired middle ears. Such measurements have, however, been made in human cadaver middle ears (Vlaming and Feenstra, 1986). In comparing the mechanics of the reconstructed cat and human middle ears, it is necessary to keep in mind the anatomical differences between these two species. In addition, it is necessary to keep in mind that there are differences in surgical techniques. Vlaming and Feenstra did not give a detailed description of their technique. For instance, no mention was made of what was done with the ossicular remnants. It is reasonable to assume, however, that a conservative approach was used in which the remnants, and hence the ossicular axis, were left intact. The results of the MSA model with an intact ossicular axis can therefore be compared with their results. For frequencies below $2 \mathrm{kHz}$, they found that displacements of the footplate's center were up to $10-20 \mathrm{~dB}$ lower (i.e., smaller 
by a factor of 3-10) than those for the ear before surgery. The present modeling results give a displacement of the footplate's centre which is virtually identical to that calculated for the normal case. In addition to the factors mentioned above, the discrepancy between experimental results and modelling results may well be due to the rigidity of the manubrium-prosthesis-stapes system in the model.

\section{Results for MFA model}

In the MFA model, the prosthesis contacts the footplate directly, rather than contacting the stapedial head as in the MSA model. Results are again reported for models both with and without an ossicular axis.

\section{With ossicular axis}

The contour patterns for eardrum displacements are qualitatively similar to the previous ones. The maximum drum displacement is $490 \mathrm{~nm}$ which is $1 \%$ larger than the value calculated for the normal middle-ear model. The displacement of the umbo is $164 \mathrm{~nm}$ which is approximately $2 \%$ larger than that calculated using the normal model.

Contours for the out-of-plane component of footplate displacement amplitude are shown in Fig. 3(b). The displacement amplitude at the centre of the footplate is $94 \mathrm{~nm}$, a value which is $1 \%$ larger than that calculated using the normal middle-ear model. The antero-posterior tilt ratio is 0.10 , which is half as large as that for the normal case. The inferosuperior tilt ratio is 0.29 , which is $12 \%$ smaller than for the normal middle-ear model. The bulge ratio is 0.055 or five times larger than for the normal case. This increase in the bulge ratio is to be expected since the prosthesis directly loads the footplate. Notice that the contours in Fig. 3(b) no longer consist of straight lines since the footplate bulges.

The in-plane motion of the footplate in this model is very similar to that of the footplate in the normal middle-ear model. The $x^{\prime}$ and $y^{\prime}$ components of translation and the angle of rotation are all within $3 \%$ of that for the normal model; the $D / R$ ratio is again less than $10^{-3}$.

\section{Absent ossicular axis}

Once again, the contours for eardrum displacements are similar to those computed for the previous models; however, as in the MSA, displacement amplitudes are larger when the axis is removed. The maximum drum displacement is 723 $\mathrm{nm}$, approximately $50 \%$ larger than for the MFA model with an intact axis of rotation. The displacement of the umbo is $329 \mathrm{~nm}$, which is about twice as large as the value computed with an intact axis.

The out-of-plane component of displacement is $122 \mathrm{~nm}$ at the center of the footplate, about 30\% larger than with an axis. The antero-posterior tilt ratio is 0.32 , or about three times larger than for the model with an axis. The inferosuperior tilt ratio is 0.15 , approximately half as large as for the MFA model with an axis. These changes are similar to those due to removing the axis from the MSA model. The bulge ratio has a value of 0.064 which is $20 \%$ larger than for the model with an axis.

The in-plane behavior can again be described by a rigidbody motion $\left(D / R\right.$ ratio is $\left.6.6 \times 10^{-4}\right)$. The $x^{\prime}$ component of translation is $16 \%$ smaller than with an axis, having a value of $57 \mathrm{~nm}$. As in the MSA model without an axis, the $y^{\prime}$ component is negative, with a value of $-61 \mathrm{~nm}$. Also as in that case, the angle of rotation is clockwise, having a value of $-8.1 \mu \mathrm{rad}$.

\section{Experimental data}

Vlaming and Feenstra (1986) did not perform any experiments with the MFA, but they did use the TORP (total ossicular replacement prosthesis), which is similar to the MFA. They found that, for frequencies below $2 \mathrm{kHz}$, displacements of the footplate's center were similar to what they had measured pre-operatively, consistent with present modeling results.

\section{Parameter variations}

Previous modeling results indicate that for frequencies below $1 \mathrm{kHz}$ the mechanical behavior of the normal cat eardrum is primarily determined by its stiffness and thickness, curvature and conical shape, and the degree of anisotropy (Funnell and Laszlo, 1978). Eardrum boundary conditions, Poisson's ratio, ossicular loading, and air loading were found to be less important. It is expected that the present models will show similar degrees of sensitivity to these eardrum parameters. In this section, the effects of varying several footplate and annular-ligament parameters are presented. For simplicity and brevity, the effects of parameter variations on footplate displacements are presented only for the MFA model with no ossicular axis. Since the prosthesis was assumed to be rigid, it was not necessary to vary its material properties.

\section{Variation of Young's modulus}

The Young's modulus of the footplate was varied from

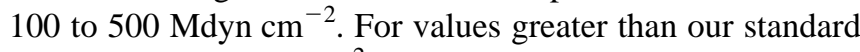
value of $200 \mathrm{Mdyn}^{-2}$, displacements of the footplate remain approximately constant. When the Young's modulus is decreased to half of the standard value, displacements of points on the rim remain approximately the same but the bulge ratio increases by $75 \%$.

The in-plane motion of the footplate does not change significantly as the Young's modulus is varied. The $x^{\prime}$ component of translation decreases by only 5\% when the Young's modulus is reduced to $100 \mathrm{Mdyn}^{-2}$; the magnitude of the $y^{\prime}$ component decreases by even less $(1 \%)$. As the Young's modulus is increased to $500 \mathrm{Mdyn} \mathrm{cm}^{-2}$, the $x^{\prime}$ component of translation increases by $2.5 \%$, and the magnitude of the $y^{\prime}$ component increases by only $0.5 \%$. The angle of rotation does not vary at all.

\section{Variation of Poisson's ratio}

Varying the Poisson's ratio from 0.0 to 0.5 has little effect on footplate displacement. The out-of-plane displacement of the central point does not change at all when the Poisson's ratio is reduced to zero, and it decreases by only $0.8 \%$ when the Poisson's ratio is increased to 0.5 .

The $x^{\prime}$ component of translation does not change when the Poisson's ratio is reduced to zero, and it increases by 


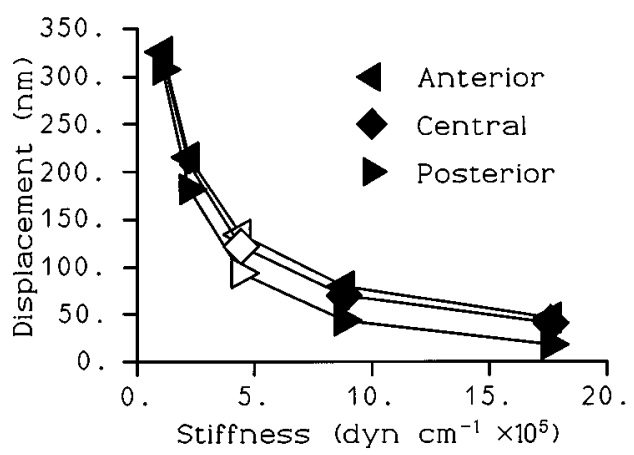

(a)

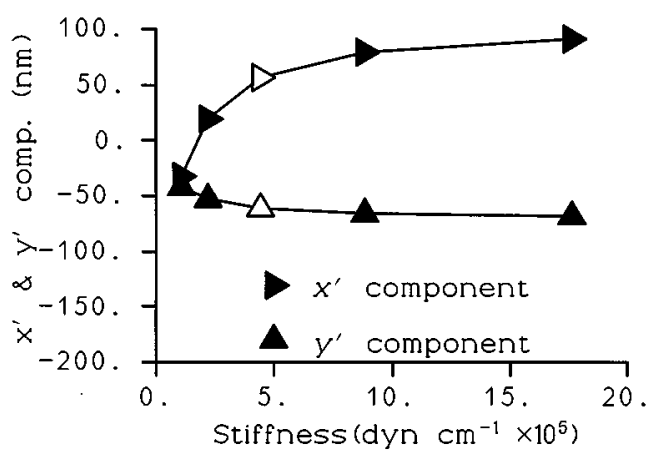

(b)

FIG. 4. Variation of out-of-plane annular-ligament stiffness. Effects on (a) out-of-plane component of displacement and (b) $x^{\prime}$ and $y^{\prime}$ components of translation. The out-of-plane components of displacement for the anterior, central and posterior points of the footplate are plotted. The input is a pure tone of $100 \mathrm{~dB}$ SPL.

only $0.4 \%$ when the Poisson's ratio is increased to 0.5 . The $y^{\prime}$ component of translation and the angle of rotation do not change as the Poisson's ratio is varied.

\section{Variation of footplate thickness}

The thickness of the central portion of the footplate was varied from 10 to $50 \mu \mathrm{m}$. For thicknesses greater than our standard value of $20 \mu \mathrm{m}$, out-of-plane footplate displacements remain approximately constant. However, for a 10$\mu \mathrm{m}$-thick footplate the displacements of the anterior and posterior ends become approximately equal, indicating that tilting about the infero-superior axis is negligible, while displacements of the inferior and superior ends are unchanged.

A $20-\mu \mathrm{m}$-thick footplate bulges only a little, and increases in the thickness above this value reduce the amount of bulging to practically zero. Decreasing the thickness below $20 \mu \mathrm{m}$, however, makes the footplate much less rigid and more prone to bulging. Changes in the thickness of the footplate have a more pronounced effect on its rigidity than do changes in its Young's modulus alone. For example, cutting the thickness in half from 20 to $10 \mu \mathrm{m}$ increases the bulge ratio by a factor of 4 , whereas cutting the Young's modulus in half increases it by only a factor of 1.75 . This is to be expected since the bending stiffness of a simple plate is proportional to the cube of its thickness, whereas it is only directly proportional to its Young's modulus.

Just as for the out-of-plane component of displacement, increasing the thickness of the footplate above $20 \mu \mathrm{m}$ has little effect on its in-plane displacements. When the thickness is decreased from 20 to $10 \mu \mathrm{m}$, the $x^{\prime}$ component of translation decreases by $28 \%$; the magnitude of the $y^{\prime}$ component increases by only 5\%; and the angle of rotation increases by $13 \%$. The $D / R$ ratio increases by a factor of 3 but is still only $1.5 \times 10^{-3}$, so the in-plane motion of the footplate can still be described by a rigid-body motion.

\section{Variation of annular-ligament stiffness}

As described above, the effects of the annular ligament on the displacements of the footplate are represented by outof-plane and in-plane springs attached along the periphery of the footplate. The effects of varying the stiffness of the annular ligament were investigated by varying the out-of-plane and in-plane stiffnesses separately and then simultaneously; it is reasonable to vary the out-of-plane and in-plane stiffnesses separately since the annular ligament may be anisotropic. These stiffnesses were reduced to one-quarter and one-half of their standard values, and they were also increased to twice and four times their standard values.

a. Variation of out-of-plane stiffness. Figure 4(a) shows the out-of-plane component of displacement for the anterior, posterior, and central points of the footplate as a function of the out-of-plane stiffness of the annular ligament. Increasing the stiffness above the standard value results in a decrease in the displacement amplitudes, whereas decreasing it below the standard value results in an increase in the displacement amplitudes. Decreasing the out-of-plane stiffness by a factor of 4 increases the out-of-plane footplate displacement by a factor of about 2.6, but increases the maximal eardrum displacement by only $22 \%$.

As can be seen from Fig. 4(b), the in-plane motion of the footplate is also affected by variations in the out-of-plane stiffness of the annular ligament. As the stiffness is decreased below the normal value, the magnitudes of both the $x^{\prime}$ and $y^{\prime}$ components of translation decrease. If the stiffness is decreased far enough, the $x^{\prime}$ component changes sign and its magnitude increases. Presumably, when the out-of-plane stiffness is decreased, it is easier for the footplate to respond to the force exerted by the prosthesis by moving into and out of the cochlea than by moving sideways. Conversely, when the out-of-plane stiffness is increased, the magnitudes of the two components of in-plane translation also increase since it is now easier for the footplate to move in its own plane than to move out of its plane. The magnitude of the angle of rotation changes by $-25 \%$ or $+50 \%$ when the stiffness is increased or decreased by a factor of 4.

b. Variation of in-plane stiffness. Figure 5(b) shows how the in-plane motion of the footplate depends on the in-plane stiffness of the annular ligament. As expected, the magnitudes of the $x^{\prime}$ and $y^{\prime}$ components of translation decrease as the in-plane stiffness is increased above the standard value. The magnitude of the $y^{\prime}$ component of translation increases when the in-plane stiffness is decreased below the standard value; the $x^{\prime}$ component of translation does not change much. The magnitude of the angle of rotation be- 


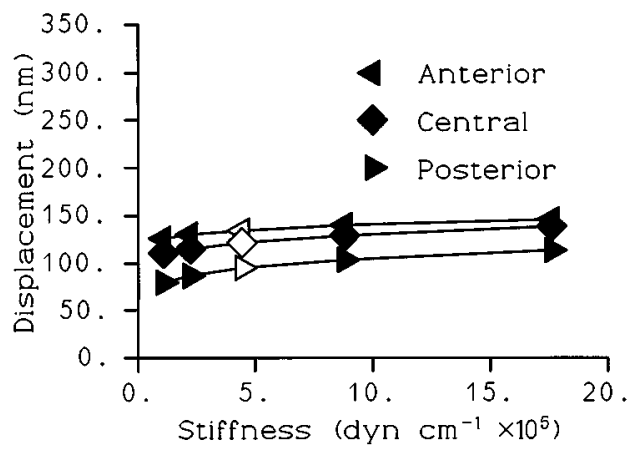

(a)

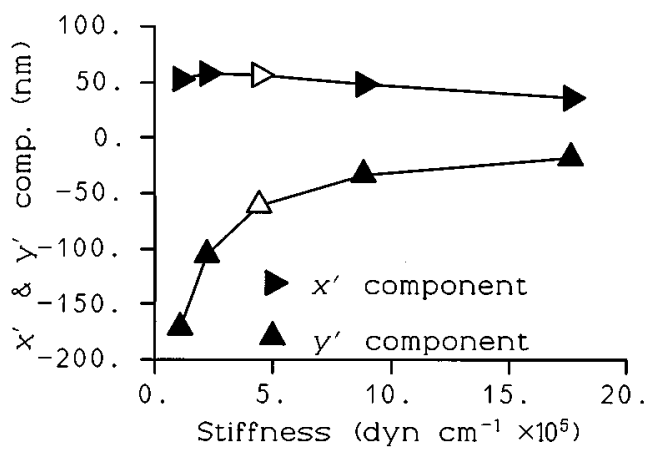

(b)

FIG. 5. Variation of in-plane annular-ligament stiffness. Effects on (a) out-of-plane component of displacement and (b) $x^{\prime}$ and $y^{\prime}$ components of translation. The input is a pure tone of $100 \mathrm{~dB}$ SPL.

comes $43 \%$ smaller when the stiffness is increased by a factor of 4 and 2.2 times larger when the stiffness is reduced by a factor of 4 below its standard value.

As shown in Fig. 5(a), increasing the in-plane stiffness of the annular ligament has some effect on the out-of-plane component of footplate displacement. The displacement at the center of the footplate increases by $13 \%$ when the inplane stiffness is increased by a factor of 4 and decreases by $10 \%$ when the in-plane stiffness is reduced to one-quarter of its value. As the in-plane stiffness is increased, there is a slightly greater tendency for the footplate to move into and out of the cochlea than to move sideways.

c. Simultaneous variation of in-plane and out-of-plane stiffnesses. Figure 6(a) shows the out-of-plane component of footplate displacement as a function of annular ligament stiffness; both the in-plane and out-of-plane stiffnesses are varied simultaneously. These curves are very similar to those obtained when the out-of-plane stiffness was varied by itself [cf. Fig. 4(a)], suggesting, as one might expect, that the outof-plane component of displacement is primarily controlled by the out-of-plane stiffness.

As shown in Fig. 6(b), the $x^{\prime}$ component of translation varies in a manner similar to that seen when the out-of-plane stiffness was varied alone. On the other hand, the $y^{\prime}$ component varies in a manner similar to that seen when the in-plane component was varied by itself. The magnitude of the angle of rotation becomes $75 \%$ smaller when the stiffness is in- creased by a factor of 4 and 2.5 times larger when the stiffness is reduced by a factor of 4 .

\section{E. Effects of prosthesis location}

It is possible to position the prosthesis at various points along the manubrium in the MFA and MSA. In the simulations presented above, the prosthesis was positioned near the upper end of the manubrium (i.e., farthest away from the umbo). In this section, the effects of positioning the prosthesis closer to the umbo are explored. Moving the prosthesis closer to the umbo does not significantly alter the mechanical behavior of the system, both with and without an ossicular axis. As an example of the changes, consider the MFA model with no ossicular axis: Moving the prosthesis down to the umbo results in a decrease of only $0.8 \%$ in the out-ofplane component of displacement of the center of the footplate [shown by the diamond symbols in Fig. 7(a)]. The inplane displacements do not change significantly either: the $x^{\prime}$ component of translation decreases by $0.9 \%$, the $y^{\prime}$ component by $0.7 \%$; and the angle of rotation decreases by $2.5 \%$.

This lack of change is expected since the manubrium and prosthesis in the model effectively act as a single rigid body, and this is consistent with the observations of Tonndorf and Pastaci (1986). They measured hearing sensitivity in anesthetized cats over a frequency range of $10 \mathrm{~Hz}$ to $15 \mathrm{kHz}$ after surgically repairing the ossicular chain with a

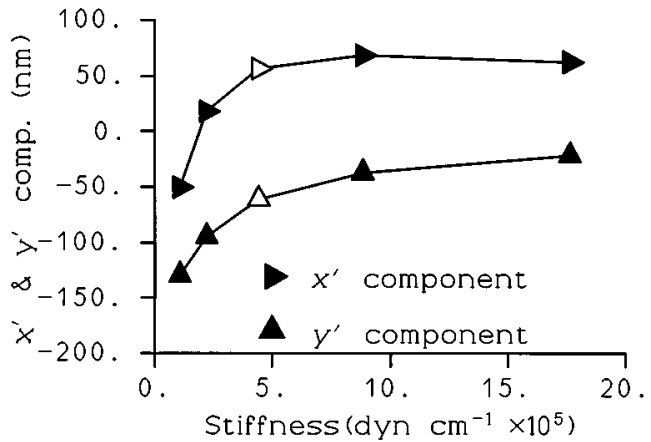

(b)

FIG. 6. Variation of total (both in-plane and out-of-plane) annular-ligament stiffness. Effects on (a) out-of-plane component of displacement and (b) $x^{\prime}$ and $y^{\prime}$ components of translation. The input is a pure tone of $100 \mathrm{~dB}$ SPL. 

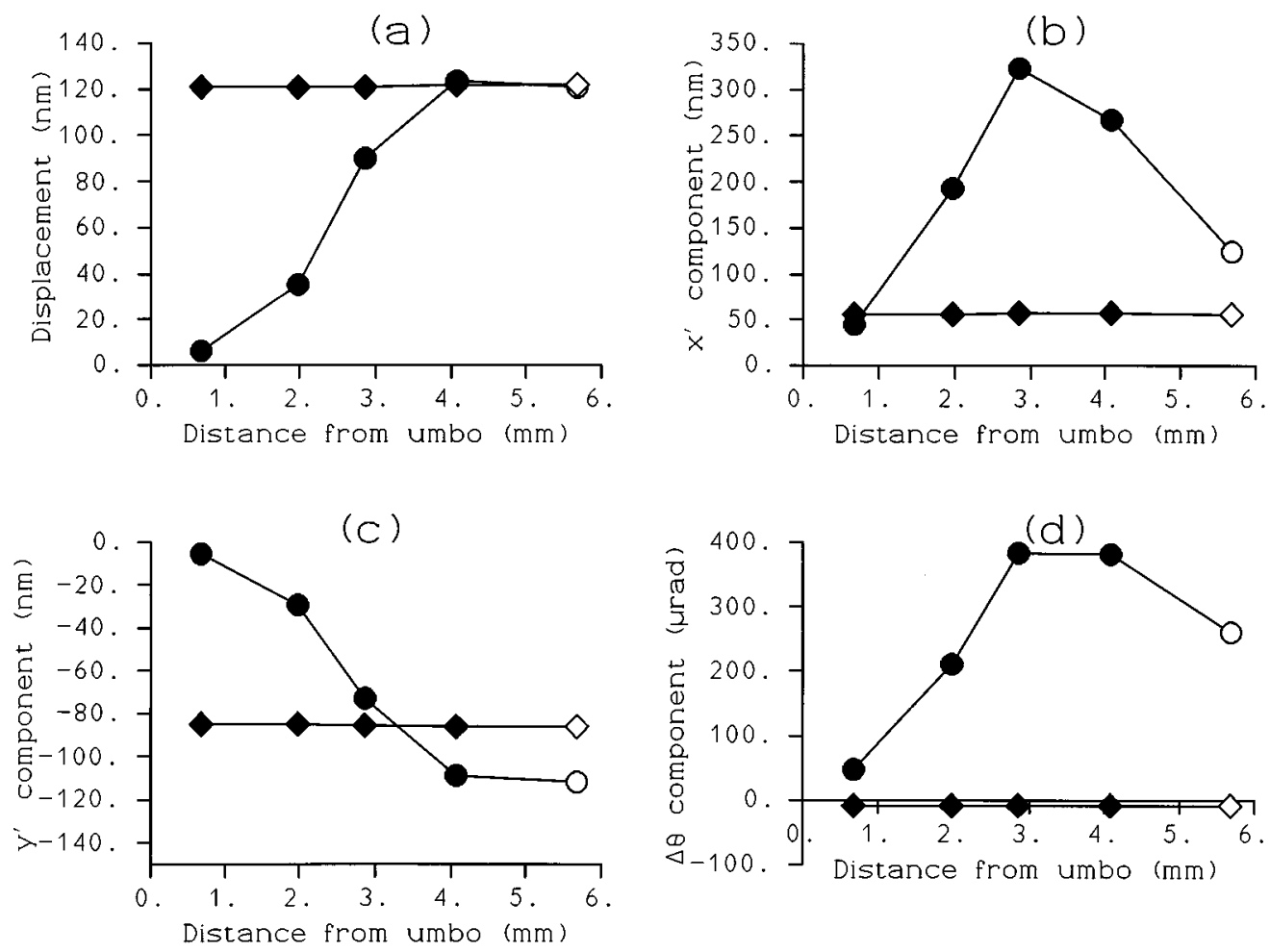

FIG. 7. Effects of positioning the prosthesis at various locations along the manubrium in the MFA model without an ossicular axis. (a) Out-of-plane component of footplate displacement at its centre versus the distance along the manubrium from the umbo, (b) $x^{\prime}$ component of translation, (c) $y^{\prime}$ component of translation, and (d) angle of rotation. The diamonds ( ) represent data for rigid joints (a brick element was used for the prosthesis), while the circles $(\bullet)$ represent data for pin joints (a truss element was used). The unfilled symbols indicate the normal location of the prosthesis at the upper end of the manubrium. The input is a pure tone of $100 \mathrm{~dB}$ SPL.

prosthesis running from the manubrium to the footplate (i.e., an MFA). The prosthesis was positioned at various points along the manubrium. They reported that the position of the prosthesis along the manubrium did not affect hearing sensitivity "... as long as the connection between the malleus and the rod [prosthesis] was made inflexible." No results were reported for flexible joints.

To quantitatively investigate the effects of flexible joints, consider an extreme case where pin joints are assumed, but the prosthesis is still rigid. Pin joints are defined as being flexible in that they cannot resist bending moments, but they are capable of transmitting tensile forces. In the SAP IV finite-element program, a rigid prosthesis with pin joints can be modeled using a rigid truss element. The circles in Fig. 7(a) show the out-of-plane displacements at the center of the footplate for the MFA model without an ossicular axis as the truss element is positioned at various locations along the manubrium. Clearly, the position of the prosthesis is important if the joints are flexible. The out-of-plane displacements of the footplate, for both the model with rigid joints and the one with pin joints, are approximately equal near the top of the manubrium (i.e., away from the umbo); however, whereas the displacements with rigid joints remain constant as the prosthesis is positioned closer to the umbo, those with pin joints decrease. The antero-posterior and the inferosuperior tilt ratios remain constant as the prosthesis is positioned at various locations along the manubrium for both kinds of joints, but are $38 \%$ higher and 4.6 times higher, respectively, with pin joints than with rigid joints. The bulge ratio varies by less than $5 \%$ about a mean value of 0.0045 which is $30 \%$ less than with rigid joints.

The in-plane motion of the footplate also depends on prosthesis position when pin joints are assumed [see Fig. 7(b) and (c)]. The $x^{\prime}$ component of translation is approximately equal to that for rigid joints when the prosthesis is located near the umbo. It first increases when the prothesis is moved away from the umbo, but then decreases when the prosthesis reaches the upper half of the manubrium. The magnitude of the $y^{\prime}$ component increases as the prosthesis is moved away from the umbo. It is smaller than that with rigid joints when the prosthesis is located on the bottom half of the manubrium, but becomes larger when the prosthesis is located on the top half. The angle of rotation is positive (i.e., in the counterclockwise direction) and increases as the prosthesis is moved away from the umbo, only decreasing slightly near the top of the manubrium, compared with a constant and negative angle of rotation for rigid joints.

\section{CONCLUSIONS}

A finite-element model of the normal cat middle ear was developed by adding explicit representations of the footplate and cochlear load to an existing model of the cat eardrum. The model was then modified to simulate the effects of middle-ear surgery. Two surgical techniques were modeled, the malleus-stapes assembly (MSA) and the malleusfootplate assembly (MFA). The models are valid for frequencies below $1 \mathrm{kHz}$ and for physiological sound levels. 
The inclusion of explicit models of the footplate and cochlear load in our eardrum model resulted in calculated displacement amplitudes which are closer to experimental data for the eardrum in the normal case. In our previous models, the in-plane stiffness of the annular ligament and the orientation of the footplate had been ignored in computing an equivalent cochlear load acting on the eardrum (Funnell and Laszlo, 1978), thus resulting in larger eardrum and umbo displacements than found using the present model.

The out-of-plane (or normal) component of displacement at the center of the footplate in the normal middle-ear model was found to be similar to that determined experimentally. The component of footplate displacement normal to its plane constitutes the mechanical input to the cochlea and, for a rigid footplate, the displacement of the center is directly proportional to the volume displacement of the cochlear fluid.

In addition to moving in a direction normal to its plane, the footplate in the normal model was found to tilt and move in its plane. Since the malleus, incus, and stapedial crura are effectively rigid in the model, and since the incudomallear and incudostapedial joints are assumed to be rigid, rotation of the malleus and incus about the ossicular axis forces the stapes to rotate about the same axis, necessarily resulting in tilting and in-plane, as well as out-of-plane, motion of the footplate. As pointed out earlier, a flexible incudostapedial joint may permit rotation of the malleus and incus about the ossicular axis without necessarily forcing the stapes to rotate about the same axis. It is interesting to note, however, that modeling the connections between the prosthesis and the bones in the MFA model as pin joints actually resulted in an increase in the amount of tilting and in-plane motion of the footplate. Direct modeling of a flexible incudostapedial joint for the normal case should clarify the issue.

For the MSA model with an intact ossicular axis, the out-of-plane component of footplate displacement was virtually identical to that calculated for the normal case, while for the MFA model with an intact axis it was slightly larger due to bulging of the footplate. Removal of the ossicular axis in both the MSA and the MFA models resulted in footplate displacements which were $30 \%$ and $50 \%$ larger, respectively, than normal. In this work, two extreme post-operative conditions of the ossicular axis were explored: one with an intact axis and the other without. It is possible that after surgery the axis may be intact, but shifted in position and orientation. Since in the present study the middle-ear ligaments suspending the ossicular chain are not directly modeled, it is not possible to predict how forces exerted by the prosthesis affect the ligaments and hence the post-operative position and orientation of the ossicular axis.

The effects of parameter variations on the displacements of the footplate were investigated for one configuration of the model. Displacements of the footplate were found to be sensitive to its Young's modulus and thickness but not to its Poisson's ratio. Footplate displacements were also found to be sensitive to the stiffness of the annular ligament. Significant bulging of the footplate was found to occur when either its Young's modulus or its thickness was reduced below the standard value, suggesting that in some individuals, it may not be suitable to apply a prosthesis directly to the centre of the footplate as in the MFA. In such cases, it may be necessary to design a prosthesis that symmetrically loads the thicker rim of the footplate, resulting in reduced bulging of the footplate as in the normal middle ear.

The position of the prosthesis along the manubrium in both the MFA and the MSA models, with or without an ossicular axis, does not affect the mechanical behavior of the footplate as long as the bones, prosthesis, and joints are all rigid. When the joints were made completely flexible, however, the out-of-plane displacements of the footplate changed dramatically: The displacements were reduced as the prosthesis was positioned further down the manubrium. This suggests that with rigid joints, which may be obtained by using glue to secure the prosthesis to the bones, a surgeon could position the prosthesis anywhere along the manubrium while still obtaining maximal volume displacement of the cochlear fluid. With flexible joints, however, careful positioning of the prosthesis along the manubrium would be required to obtain similar results.

\section{ACKNOWLEDGMENTS}

This work was supported by the Medical Research Council of Canada and the Natural Sciences and Engineering Research Council of Canada. We thank Dr. M. D. Schloss, Chair of the Department of Otolaryngology, McGill University, for providing insight on middle-ear surgery, and $\mathrm{J}$. Lauzière for editing the manuscript.

Austin, D. F. (1971). “Ossicular reconstruction,'” Arch. Otolaryngol. 94, 525-535.

Bathe, K.-J., Wilson, E. L., and Peterson, F. E. (1974). "SAP IV. A structural analysis program for static and dynamic response of linear systems,", Report No. EERC 73-11, University of California, Berkeley.

Decraemer, W. F., Khanna, S. M., and Funnell, W. R. J. (1991). “Malleus vibration mode changes with frequency,' Hear. Res. 54, 305-318.

Funnell, S. M. (1989). "An Approach to Finite-Element Modelling of the Middle Ear,' Master's thesis, McGill University, Montréal.

Funnell, W. R. J. (1983). "On the undamped natural frequencies and mode shapes of a finite-element model of the cat eardrum,' J. Acoust. Soc. Am. 73, 1657-1661.

Funnell, W. R. J., and Laszlo, C. A. (1978). "Modeling of the cat eardrum as a thin shell using the finite-element method,' J. Acoust. Soc. Am. 63, 1461-1467.

Funnell, W. R. J., and Laszlo, C. A. (1982). "A critical review of experimental observations on ear-drum structure and function,", ORL 44, 181205.

Funnell, W. R. J., Decraemer, W. F., and Khanna, S. M. (1987). "'On the damped frequency response of a finite-element model of the cat eardrum,', J. Acoust. Soc. Am. 81, 1851-1859.

Funnell, W. R. J., Khanna, S. M., and Decraemer, W. F. (1992). "On the degree of rigidity of the manubrium in a finite-element model of the cat eardrum,"' J. Acoust. Soc. Am. 91, 2082-2090.

Guinan, J. J., Jr., and Peake, W. T. (1967). "Middle-ear characteristics of anesthetized cats,' J. Acoust. Soc. Am. 41, 1237-1261.

Khanna, S. M. (1970). “A Holographic Study of Tympanic Membrane Vibrations in Cats,' Ph.D. thesis, City University of New York, New York.

Lesser, T. H. J., Williams, K. R., and Blayney, A. W. (1991). “Mechanics and materials in middle ear reconstruction," Clin. Otolaryngol. 16, 29-32.

Lynch, T. J., III, Nedzelnitsky, V., and Peake, W. T. (1982). “'Input impedance of the cochlea in cat,"' J. Acoust. Soc. Am. 72, 108-130.

Pang, X. D., and Peake, W. T. (1986). "How do contractions of the stapedius muscle alter the acoustic properties of the ear?' in Peripheral Auditory Mechanisms. Proceedings, 1984, edited by J. B. Allen, J. L. Hall, A. 
Hubbard, S. Neely, and A. Tubis (Springer-Verlag, New York), pp. 3643.

Peake, W. T., Rosowski, J. J., and Lynch, T. J., III (1992). "Middle-ear transmission: acoustic versus ossicular coupling in cat and human," Hear. Res. 57, 245-268.

Tonndorf, J., and Khanna, S. M. (1971). "The tympanic membrane as a part of the middle ear transformer," Acta Otolaryng. 71, 177-180.

Tonndorf, J., and Pastaci, H. (1986). "Middle ear sound transmission: A field of early interest to Merle Lawrence," Am. J. Otolaryngol. 7, 120129.

Vartiainen, E., and Nuutinen, J. (1992). "Long-term hearing results of onestage tympanoplasty for chronic otitis media," Eur. Arch. Otorhinolaryngol. 249, 329-331.

Vlaming, M. S. M. G. (1987). "Middle ear mechanics studied by laser
Doppler interferometry," Ph.D. thesis, Technische Universiteit Delft, Delft.

Vlaming, M. S. M. G., and Feenstra, L. (1986). "Studies on the mechanics of the reconstructed human middle ear," Clin. Otolaryngol. 411-422.

Wada, H., Tetsuro, M., and Kobayashi, T. (1992). "Analysis of dynamic behaviour of human middle ear using a finite-element method,' J. Acoust. Soc. Am. 92, 3157-3168.

Wada, H., Toshimitsu, K., and Tetsuro, M. (1990). "Optimal design of columella-type ceramic artificial auditory ossicle (Determination of ossicle configuration by theoretical analysis)," Trans. Japan Soc. Mech. Eng. 56, $1435-1439$.

Williams, K. R., and Lesser, T. H. J. (1992). "A dynamic and natural frequency analysis of the Fisch II spandrel using the finite element method,' Clin. Otolaryngol. 17, 261-270. 